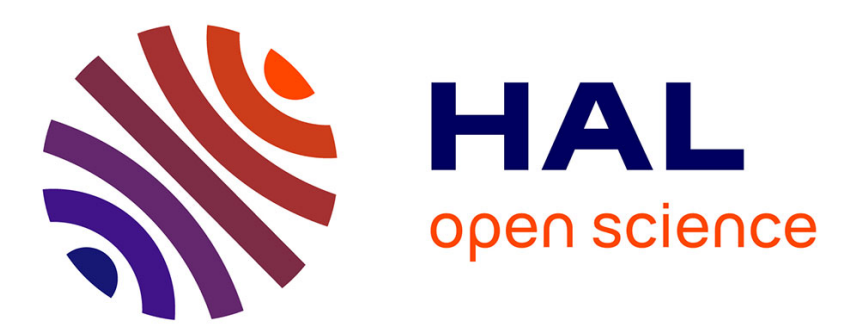

\title{
The effect of thermal boundary conditions on dynamos driven by internal heating
}

K. Hori, J. Wicht, U.R. Christensen

\section{To cite this version:}

K. Hori, J. Wicht, U.R. Christensen. The effect of thermal boundary conditions on dynamos driven by internal heating. Physics of the Earth and Planetary Interiors, 2010, 182 (1-2), pp.85. 10.1016/j.pepi.2010.06.011 . hal-00675796

\section{HAL Id: hal-00675796 https://hal.science/hal-00675796}

Submitted on 2 Mar 2012

HAL is a multi-disciplinary open access archive for the deposit and dissemination of scientific research documents, whether they are published or not. The documents may come from teaching and research institutions in France or abroad, or from public or private research centers.
L'archive ouverte pluridisciplinaire HAL, est destinée au dépôt et à la diffusion de documents scientifiques de niveau recherche, publiés ou non, émanant des établissements d'enseignement et de recherche français ou étrangers, des laboratoires publics ou privés. 


\section{Accepted Manuscript}

Title: The effect of thermal boundary conditions on dynamos driven by internal heating

Authors: K. Hori, J. Wicht, U.R. Christensen

PII:

S0031-9201(10)00131-7

DOI: doi:10.1016/j.pepi.2010.06.011

Reference: PEPI 5303

To appear in: Physics of the Earth and Planetary Interiors

Received date: 23-3-2010

Revised date: 2-6-2010

Accepted date: 22-6-2010

Please cite this article as: Hori, K., Wicht, J., Christensen, U.R., The effect of thermal boundary conditions on dynamos driven by internal heating, Physics of the Earth and Planetary Interiors (2010), doi:10.1016/j.pepi.2010.06.011

This is a PDF file of an unedited manuscript that has been accepted for publication. As a service to our customers we are providing this early version of the manuscript. The manuscript will undergo copyediting, typesetting, and review of the resulting proof before it is published in its final form. Please note that during the production process errors may be discovered which could affect the content, and all legal disclaimers that apply to the journal pertain. 


\title{
The effect of thermal boundary conditions on dynamos driven by internal heating
}

\author{
K. Hori ${ }^{*, a}$, J. Wicht ${ }^{\mathrm{a}}$, U.R. Christensen ${ }^{\mathrm{a}}$ \\ ${ }^{a}$ Max-Planck Institute for Solar System Research, Max-Planck-Strasse 2, 37191 \\ Katlenburg-Lindau, Germany
}

\begin{abstract}
The early dynamos of Mars and Earth probably operated without an inner core being present. They were thus exclusively driven by secular cooling and radiogenic heating which can both be modeled by homogeneously distributed heat sources. Some previous dynamo simulations that explored this driving mode found dipole dominated magnetic fields, while other reported multipolar configurations. Since these models differed both in the employed outer thermal boundary conditions and in the size of the inner core, which was still retained for practical reasons, the cause for the variation in field geometry remained unclear. Here we investigate this issue and find that strong dipole dominated fields are preferred for fixed heat flux conditions whereas weaker multipolar fields are typical for fixed temperature conditions. The size of the inner core, on the other hand, proved to be of minor influence. The stronger dipolar fields for fixed heat flux conditions promote larger convective structures. Since the mantle of the terrestrial planets controls the heat flux rather than the temperature at the core-mantle boundary, our re-
\end{abstract}

\footnotetext{
*Corresponding author. Tel.:+49-5556-979-379; Fax: +49-5556-979-240.

Email address: hori@mps.mpg.de (K. Hori)
} 
sults suggest that the early dynamos of Mars and Earth would have produced dipole dominated magnetic fields.

Key words: MHD dynamo, internal heating, thermal boundary condition, inner core, paleo-magnetic field

\section{Introduction}

According to thermal evolution models, Earth had no solid inner core until about 1-2 billion years ago (Labrosse, 2003; Nimmo, 2007). When an inner core is absent, a dynamo must be driven by volumetric secular cooling and possibly radiogenic internal heating, whereas the present geodynamo is through to be largely driven by a buoyancy flux from below, arising from the release of latent heat and compositional enrichment associated with inner core freezing (e.g. Stevenson et al., 1983; Labrosse, 2003; Nimmo, 2007). The early Martian dynamo probably also operated without an inner core and was driven by secular cooling (e.g. Stevenson, 2001). If a growing inner core had been present, it is difficult to understand why the Martian dynamo stopped to operate approximately 4.1 billion years ago (Lillis et al., 2008). In the absence of an inner core the dynamo could have stopped because the declining heat-flow has led to a subadiabatic temperature gradient in the fluid core.

The presence or absence of an inner core affects the dynamo in various ways - through its electrical conductivity, through its influence on the geometry of the flow in the outer core and by its role as a buoyancy source. Inner core conductivity has been proposed as being essential for stabilizing the dipole field against too frequent reversals (e.g. Hollerbach \& Jones, 1993). 
Numerical simulations comparing cases with a conducting and an insulating inner core (Wicht, 2002) and with or without an inner core (Sakuraba \& Kono, 1999) suggest that the differences for the observable field outside the core are small.

The geometrical effect arises because the inner core represents an obstacle to the preferred pattern of convection, which consists of nearly-geostrophic convection columns aligned with the rotation axis (e.g. Busse, 2002). This effect occurs also for non-magnetic convection. Dormy et al. (2004) investigated the onset of thermal convection with homogeneous heat sources in the inner and outer core. They find that the structure of convection at onset hardly depends on the inner core radius, provided it is less than approximately $45 \%$ of the core radius (so-called thick shell regime), and that the convection is similar to that in a full sphere.

Perhaps the most profound difference between dynamos with and without an inner core comes from the different distribution of buoyancy sources. In the absence of an inner core, the lack of the buoyancy flux associated with its growth implies that convection is weaker and Ohmic dissipation lower than for the present Earth's core. Furthermore, the different distribution of sources and sinks of buoyancy flux may lead to different morphologies of the magnetic field. Here we separate the question of the existence of an inner core from that of the mode of driving convection in the fluid core. We focus on dynamos in a thick shell where the buoyancy sources are volumetrically distributed and where the outer boundary represents the sink for the buoyancy flux. This represents convection that is driven by internal heat sources or secular cooling or a combination of both. Hereafter we refer to this scenario as 
internal heating and we use the term basal heating when the buoyancy source is located at the inner core boundary.

Previous dynamo simulations proposed that dynamos driven by significant degrees of internal heating produce different field properties, compared to those with no internal heating. Busse and co-workers (e.g. Grote et al., 2000; Busse, 2002) studied the field morphology for dynamos driven by a combination of internal heating and basal heating, mostly with stress-free mechanical boundary conditions. Depending on the various control parameters, they found a diversity of field morphologies, comprising dipolar and non-dipolar solutions with various field geometries. Dynamos with dipoledominated fields are more commonly found with pure basal heating and noslip mechanical condition (e.g. Christensen et al., 1999). Directly comparing cases of internal heating, of basal heating and of compositional convection (where buoyancy sources at the inner boundary are balanced by volumetric sinks) for the case of no-slip conditions, Kutzner \& Christensen (2000, 2002) found that internal heating favours solutions with non-dipolar magnetic fields at the same control parameter values where dipolar dynamos prevail for the other modes of driving convection.

For dynamos with an imposed temperature contrast Christensen \& Aubert (2006) found that a local Rossby number, which is a measure for the ratio between inertial and Coriolis forces, controls the field structure. At low values the solution is dipolar and at high values (approximately $>0.12$ ) the field at the outer boundary of the dynamo is dominated by higher multipoles. Olson \& Christensen (2006) showed that the dipolar-multipolar transitions as a function of the local Rossby number is less sharp for internally heated 
dynamos than it is for models with fixed temperature contrast and that nondipolar solutions persist to lower values of the local Rossby number. The dipole moment was found to be generally weaker at the same value of the buoyancy flux in the internally heated case.

In contrast to these earlier results, Aubert et al. (2009) found in recent dynamo simulations relatively small differences, at a given value of the convective power, in terms of the magnetic field strength and of the relative dipole contribution to the field at the outer boundary between dynamos with internal heating, basal heating or compositional convection. Their models for internally heated dynamos differed in two respects from earlier ones. Previous models employed a condition of fixed temperature on the outer boundary, whereas Aubert et al. (2009) impose a fixed homogeneous heat flux, which is a more natural condition for dynamos in terrestrial planets. The difference in the thermal boundary condition can have a significant influence on the pattern of convection and the properties of the magnetic field (Sakuraba \& Roberts, 2009). Furthermore, most earlier studies used the present radius of the Earth's inner core, $35 \%$ of the core radius, whereas Aubert et al. (2009) reduced the inner core size to 1 - $5 \%$. Most internally-heated models retained a passive inner core, because the current spectral dynamo codes usually only allow to simulate a spherical shell but not a full sphere. The differences between the results of Aubert et al. (2009) and those of Kutzner \& Christensen $(2000,2002)$ and Olson \& Christensen (2006) could be caused by the thermal boundary condition or by the difference in inner core size.

Roberts \& Glatzmaier (2001) explored three models related to the past, present and future geodynamo with different sizes of the inner core, using 
heat flux conditions on the boundaries. In their model with a small inner core the dipole was found to be more dominant than in the other cases. However, even their model with a small inner core was mainly driven by basal heating, because the smaller surface area of the inner core was balanced by a much faster growth in radius than at present. For this reason there is a strong difference between the situation without an inner core and that with even a small inner core. The influence of the inner core size on the dynamo onset in the case of bottom heated convection was also studied by Heimpel et al. (2005).

In most MHD dynamo simulations fixed temperature conditions have been the standard (e.g. Kageyama \& Sato, 1995; Olson et al., 1999; Takahashi et al., 2008a). Others used a heat flux boundary condition, but in many cases the emphasis was on exploring the influence of various pattern of heterogeneous heat flux distribution at the core-mantle boundary (e.g. Glatzmaier et al., 1999; Olson \& Christensen, 2002; Christensen \& Olson, 2003; Takahashi et al., 2008b). Stanley et al. (2008) proposed that a strong hemispherical dichotomy of the heat flux out of the early Martian core has led to a dynamo operating only in one hemisphere. This could explain the observed uneven distribution in the magnetization on the Martian crust observed by Mars Global Surveyor. The question whether the nature of the thermal boundary condition, fixed flux or fixed temperature, makes a fundamental differences has been addressed in a few studies only. For non-magnetic rotating convection the heat flux boundary condition favours larger scales of convection than the temperature condition near the onset of convection (Takehiro et al., 2002). Comparing dynamo models with different thermal boundary con- 
ditions, Busse \& Simitev (2006) reported no major qualitative differences. Recently, Sakuraba \& Roberts (2009) compared the effect of the boundary condition for a rapidly-rotating dynamo model (low Ekman number). They found that the heat flux condition promotes stronger magnetic fields and larger scales in the velocity and magnetic field.

The purpose of this paper is to clarify the influence of thermal boundary conditions and the size of the inner core on dynamos driven by internal heating. The inner core is kept only for technical reasons and is made passive in the sense that it is not a source of buoyancy nor is it electrically conducting. By varying its size we want to determine if its kinematic influence on the dynamo is significant. We compare dynamos with uniform temperature and uniform heat flux, respectively, for otherwise identical sets of control parameters. The model setup and the diagnostic parameters that we use to compare the results are described in Section 2. In Section 3 we demonstrate that the thermal boundary condition rather than the inner core size has a major role on the field morphology and in Section 4 we discuss the implications for the early geodynamo and the Martian dynamo.

\section{Formulation}

We model a rotating spherical shell with inner core radius $r_{\mathrm{i}}$ and outer radius $r_{\mathrm{o}}$ that is filled with an electrically conducting fluid. Convection is driven by homogeneously distributed volumetric heat sources. We solve the following dimensionless equations in the Bousinesq approximation: the heat transport equation (1), the Navier-Stokes equation (2), the induction equation (3), and the conditions for incompressible fluid and solenoidal field (4): 


$$
\begin{aligned}
\frac{\partial T}{\partial t}+\mathbf{u} \cdot \nabla T & =\frac{1}{P r} \nabla^{2} T+1, \\
E k\left(\frac{\partial \mathbf{u}}{\partial t}+\mathbf{u} \cdot \nabla \mathbf{u}\right) & +2 \hat{\mathbf{e}_{z}} \times \mathbf{u} \\
=-\nabla P & +R a^{\prime} T \frac{\mathbf{r}}{r_{\mathrm{o}}}+E k \nabla^{2} \mathbf{u}+\frac{1}{P m}(\nabla \times \mathbf{B}) \times \mathbf{B}, \\
\frac{\partial \mathbf{B}}{\partial t} & =\nabla \times(\mathbf{u} \times \mathbf{B})+\frac{1}{P m} \nabla^{2} \mathbf{B}, \\
\nabla \cdot \mathbf{u} & =0, \quad \nabla \cdot \mathbf{B}=0,
\end{aligned}
$$

where $T, \mathbf{u}, P$ and $\mathbf{B}$ are temperature, velocity, non-hydrostatic pressure and magnetic field, respectively. The fundamental scales for length, time, temperature and magnetic field are, respectively, the thickness $D=r_{\mathrm{o}}-r_{\mathrm{i}}$ of the spherical shell, the viscous diffusion time $D^{2} / \nu$ (with $\nu$ the kinematic viscosity), $\epsilon D^{2} / \nu$ (with $\epsilon$ the internal heating rate) and $(\rho \mu \lambda \Omega)^{1 / 2}$ (with $\rho$ the density, $\mu$ the magnetic permeability, $\lambda$ the magnetic diffusivity and $\Omega$ the rotation rate). The four non-dimensional control parameters are the Ekman number, Prandtl number, magnetic Prandtl number and modified Rayleigh number:

$$
E k=\frac{\nu}{\Omega D^{2}}, \quad \operatorname{Pr}=\frac{\nu}{\kappa}, \quad \operatorname{Pm}=\frac{\nu}{\lambda}, \quad R a^{\prime}=\frac{\alpha g_{o} \epsilon D^{3}}{\nu^{2} \Omega},
$$

where $\kappa, \alpha$ and $g_{o}$ are the thermal diffusivity, thermal expansivity and gravity at the outer boundary, respectively.

We impose a zero heat flux at the inner core boundary. On the outer boundary, we assume either a fixed temperature condition, $T\left(r_{\mathrm{o}}\right)=0$, or a uniform heat flux, $\partial T / \partial r\left(r_{\mathrm{o}}\right)=-q_{o}$. We refer to the former as the 'FT' condition and to the latter as the 'FF' condition. In the FF cases, the value of the heat flux at the outer boundary is in balance with the internal heating rate 


$$
4 \pi r_{\mathrm{o}}^{2} q_{o}=\frac{4}{3} \pi\left(r_{\mathrm{o}}^{3}-r_{\mathrm{i}}^{3}\right) .
$$

Note that the dimensionless heating rate density is one because of the temperature scale chosen here. When the internal heating rate is zero for an unchanged heat flux at the outer boundary (secular cooling), the solution would be identical except for a linear drift of the temperature. The velocity is forced to vanish on both boundaries (no-slip condition) and the mantle and inner core are electrically insulating and co-rotating.

Aside from the thermal boundary condition and the Rayleigh number, we vary the size of the inner core, assuming values of either 0.35 or 0.10 for the ratio

$$
\eta=r_{\mathrm{i}} / r_{\mathrm{o}}
$$

We vary the modified Rayleigh number $R a^{\prime}$ in the range of $350-2100$ for each of the four combinations of thermal boundary conditions and inner core sizes. For cases with the large aspect ratio we also study higher values of the Rayleigh number. The other parameters are fixed to $E k=10^{-4}, \operatorname{Pr}=1$, and $P m=3$. The critical Rayleigh numbers $R a_{\text {crit }}^{\prime}$ for the non-magnetic convection are 108 (201) and 109 (202) for FT and FF cases, respectively, at $\eta=0.35(0.10)$. They have been calculated by monitoring the growth or decay of weak thermal perturbations of the conductive state. The critical values are practically identical for the two thermal boundary conditions at these values of $E k$ and $P r$, in agreement with results by Busse \& Simitev (2006) and Gibbons et al. (2007), while reducing the inner core size from $\eta=0.35$ to 0.10 roughly doubles the critical Rayleigh number. In terms of the supercriticality, our simulations cover the range of $3.2-77(2.2-10)$ at $\eta=0.35(0.10)$ 
We describe the characteristic properties of the solution in terms of timeaveraged non-dimensional measures most of which have been defined in previous papers (Kutzner \& Christensen, 2002; Christensen \& Aubert, 2006). The Nusselt number measures the efficiency of heat transfer and we here use the definition

$$
N u^{*}=\frac{\Delta T_{\mathrm{S}}}{\Delta \bar{T}}=\frac{T_{\mathrm{S}}\left(r_{\mathrm{i}}\right)-T_{\mathrm{S}}\left(r_{\mathrm{o}}\right)}{\bar{T}\left(r_{\mathrm{i}}\right)-\bar{T}\left(r_{\mathrm{o}}\right)} .
$$

The overbar indicates the average over a spherical surface and the suffix $\mathrm{S}$ stands for the conductive solution.

The amplitude of the rms-velocity $u_{\mathrm{rms}}$ is given in terms of the magnetic Reynolds number

$$
R m=\frac{u_{\mathrm{rms}} D}{\lambda}
$$

A measure for the ratio between inertial and Coriolis forces is the local Rossby number

$$
R o_{\ell}=R o \frac{l_{\mathrm{u}}}{\pi}=\frac{u_{\mathrm{rms}}}{\Omega D} \frac{l_{\mathrm{u}}}{\pi}
$$

where $R o$ is the conventional Rossby number and $l_{\mathrm{u}}$ is the mean spherical harmonic degree $l$ in the kinetic energy spectrum

$$
l_{\mathrm{u}}=\frac{\sum_{l} l E_{\mathrm{kin}}^{(l)}}{E_{\mathrm{kin}}} .
$$

Christensen \& Aubert (2006) and Olson \& Christensen (2006) found that for the bottom heated dynamos with FT condition the local Rossby number controls whether the magnetic field is dominantly dipolar or multipolar.

The strength of the magnetic field in the volume is measured by the Elsasser number

$$
\Lambda=\frac{B_{\mathrm{rms}}^{2}}{\rho \mu \lambda \Omega}
$$


which equals the square of the non-dimensional field strength in our scaling. We characterise the morphology of the magnetic field on the outer boundary of the dynamo in terms of its dipolarity, defined as the time average power of the axial dipole field relative to the total power in spherical harmonic degrees up to twelve:

$$
f_{\text {dip }}^{\text {ax }}=\left(\frac{E_{\text {mag }}^{(l=1, m=0)}\left(r=r_{\mathrm{o}}\right)}{\sum_{l=1}^{12} \sum_{m=0}^{l} E_{\text {mag }}^{(l, m)}\left(r=r_{\mathrm{o}}\right)}\right)^{1 / 2} .
$$

In previous works, the dipolarity $f_{\text {dip }}$ was defined in terms of the total dipole component. Since only the axial part of the dipole has special significance, we here define the dipolarity for this component alone.

Some of our dynamos are not dominated by the dipole but exhibit various complex field structures as described below. We classify the various morphologies in terms of the spatial patterns that we observe on the outer boundary at several snapshots in each run. This classification is supported by the dominant degree $l_{\mathrm{B}}^{\mathrm{p}}$ and order $m_{\mathrm{B}}^{\mathrm{p}}$ which we obtain from the peak in the time- and radially- averaged magnetic energy spectrum. To further characterize the magnetic field, we also record time-averaged values of the relative fractions of the axisymmetric poloidal $(P)$ and toroidal $(T)$ magnetic energies in the spherical shell volume. The corresponding ratios for the kinetic energy indicate the relative contributions of zonal wind $(D)$ and meridional circulation $(M)$, respectively, to the flow. We describe the strength of each axisymmetric component in terms of the relative fraction of the total energy, because we are mostly interested in the structural differences in the flow and the magnetic field. In absolute terms, the total kinetic energy is obtained as $0.5(R m / P m)^{2}$ and the total magnetic energy is $\Lambda /(2 P m E k)$.

We use the code MagIC (e.g. Wicht, 2002), which is a modified version of 
a code originally developed by Glatzmaier (e.g. Glatzmaier, 1984) and solves the equations with a pseudo-spectral method. The horizontal resolution in terms of the maximum spherical harmonic degree and order is $L=M=$ 96, corresponding to $N_{\phi}=288$ and $N_{\theta}=144$ grid points in azimuthal and latitudinal directions, respectively. In radial direction we use $N_{r}=49$ Chebyshev polynomials for cases with $\eta=0.35$ and $N_{r}=71$ for cases with $\eta=0.10$. The two cases at the highest Rayleigh numbers and $\eta=0.35$ with FF boundary conditions required $L=M=128$ and $N_{r}=61$.

A dynamo solution with a strong dipolar field was used as initial condition in all our runs. For several cases we confirmed the uniqueness by also starting from multipolar solutions. All runs were integrated for at least two magnetic diffusion times.

\section{Results}

The numerical experiments and results are listed in Table 1 in terms of the control parameters, boundary conditions, time-averaged properties of the solutions and the classification of the field morphology. Fig. 1 and Fig. 2 summarize how the field geometry depends on the parameters. Fig. 1 shows the dependence of the dipolarity on the local Rossby number. For low $R o_{\ell}$ values (typically $R o_{\ell}<0.1$ ), the field at the outer boundary is highly dipolar for the FF boundary condition $\left(f_{\text {dip }}^{\text {ax }}>0.45\right)$ but multipolar for the FT condition $\left(f_{\text {dip }}^{\text {ax }}<0.15\right)$. The aspect ratio $\eta$ has a much smaller effect on the dipolarity than the thermal boundary condition. The discrepancy between the findings by Kutzner \& Christensen (2000) and Aubert et al. (2009) therefore likely results from the different thermal boundary condition. 
The strong influence of the thermal boundary condition is also obvious in Fig. 2 , which shows a phase diagram for the dependence of the field morphology at the outer boundary on the aspect ratio $\eta$ and on the degree of supercriticality of the Rayleigh number $R a^{\prime} / R a_{\text {crit }}^{\prime}$. The fields for the FF condition are all dominated by the axial dipole. For the FT condition the axial dipole is much weaker and the field shows different multipolar geometries, depending on $\eta$ and $R a^{\prime} / R a_{\text {crit }}^{\prime}$.

In the following subsections, we describe typical features of FT (Section 3.1) and FF (Section 3.2) simulations. We focus on the low $R o_{\ell}$ regime, where the differences are apparent, and compare four cases at $R a^{\prime}=700$, where $R o_{\ell}$ is between $0.012(\mathrm{FF}, \eta=0.10)$ and $0.042(\mathrm{FT}, \eta=0.35)$. For $R o_{\ell} \gtrsim 0.1$, all solutions are expected to be multipolar.

\subsection{Fixed temperature boundary conditions}

The fixed temperature boundary conditions promote weaker magnetic fields, with Elsasser numbers between 0.1 and 4.0, and complex non-dipolar field geometries, with dominant spherical degrees larger than one (Table 1). Non-axisymmetric field contributions dominate at the outer boundary as well as throughout the volume in all FT cases, with the exception of the model at $R a^{\prime}=700$ and $\eta=0.10$ where the axisymmetric quadrupole $(l=2, m=0)$ is most prominent at the outer boundary. Figure 3 (thin lines) shows the volume averaged energy spectra at $R a^{\prime}=700$ for both aspect ratios. Harmonic orders $m=1$ dominate in the two cases. Axisymmetric contributions are small and account for not more than $7 \%$ of the total magnetic energy $(P+T)$. This increases to $20 \%$ for the quadrupolar case at $\eta=0.10$ and $R a^{\prime}=700$. 
A comparison of the spectra for the two aspect ratios reveals that the thinner shell at $\eta=0.35$ promotes smaller magnetic length scales: the maximum at low degree is less pronounced than at $\eta=0.10$ and more energy is concentrated at degrees beyond $l=15$, resulting in a rather flat spectrum. This becomes even more pronounced in the cases with large Rayleigh numbers $R a^{\prime}=2100$ and 4200 at $\eta=0.35$, where the spectra show a rather broad maximum between $l=7$ and $l=20$ (not shown). Table 1 illustrates that the relative contribution of axial dipole $f_{\text {dip }}^{\text {ax }}$ slightly increases with the local Rossby number $R o_{\ell}$ for either aspect ratio, though it stays small and renders all FT cases as non-dipolar.

Figure $4 \mathrm{a}$ and $\mathrm{b}$ show typical configuration of the radial field at the outer boundary for the two FT cases at $R a^{\prime}=700$. Fig. 4a illustrates the hemispherical geometry of the field found at $\eta=0.35$, where the strong field patches predominantly concentrate in one hemisphere. The hemispherical field can be described as a superposition of fields with dipolar and quadrupolar symmetries, each of which does not require an equatorial asymmetry of the flow (which we do not observe). Fig. 4b shows the equatorially symmetric field we find at $\eta=0.10$. The axisymmetric magnetic fields depicted in Figure 5 confirm that either field characteristic, hemispherical or equatorially symmetric, prevails throughout the shell. A configuration dominated by an equatorially symmetric octpole $(l=3, m=1)$ is observed at an aspect ratio of $\eta=0.35$ and a low Rayleigh number of $R a^{\prime}=350$ (not shown here).

Grote et al. (2000) find hemispherical dynamos at parameters close to the ones employed here, at $P m \sim 3, P r=1$ and $E k \sim 10^{-4}$, with the stress-free boundary conditions. They also report that dipolar dynamos are recovered 
at larger magnetic Prandtl numbers, which we have not investigated so far. Further we observe oscillatory behaviours of the field, as reported in Busse \& Simitev (2006). For example, in some models the activity switches between hemispheres. The quadrupolar field shows a periodic oscillation in which structures in the axisymmetric poloidal and toroidal fields propagate poleward in each hemisphere.

The fluid motion is dominated by non-axisymmetric contributions. The energies in the axisymmetric flows $(D+M)$ amount to only $5 \%$ and $8 \%$ of the total kinetic energy in the cases of $\eta=0.35$ and $\eta=0.10$, respectively. Figure 6 illustrates contours of the $z$-component of the vorticity in the equatorial plane. The convection cells concentrate in the outer regions of the shell, where the conductive temperature gradient is steeper than deep inside of the shell. This concentration of convection cells on the outer regions is obvious in the case with a small inner core, in which the Rayleigh number is closer to the critical value than that in the case with a large inner core. The helicity, $\overline{\mathbf{u} \cdot(\nabla \times \mathbf{u})}$, is therefore concentrated near the outer boundary (Figure $5 \mathrm{c}$ and f). The kinetic energy spectra show broad peaks at $8 \lesssim m \lesssim 11$ and $5 \lesssim m \lesssim$ 9 for $\eta=0.35$ and 0.10 (thin solid lines in Figure $7 \mathrm{a}$ and $\mathrm{b}$ ), respectively, and little dependence on the Elsasser number. We cannot observe the effect that Lorentz forces promote larger flow scales, which some magnetoconvection simulations found for $\Lambda>\mathcal{O}\left(E k^{1 / 3}\right)$ or others for $\Lambda>\mathcal{O}(1)$ (Jones (2007) and Wicht et al. (2009) for recent overviews).

Figure 8 shows snapshots of the axisymmetric temperature distribution, zonal flow and axisymmetric meridional circulation. Plume-like convection is shown to set in inside the tangent cylinder (hereafter referred as TC) at 
Rayleigh numbers which are a few times the critical Rayleigh number. Once it has started, it can transport heat effectively inside the TC as well. This effect is responsible for the latitudinal dependence of the zonally averaged temperature in the case with $\eta=0.35$ and $R a^{\prime}=700$ (Fig. 8a). The plumes drive a strong meridional circulation (Fig. 8c) and are also responsible for the strong thermal winds arising inside the TC (Fig. 8b). For $\eta=0.10$, the TC occupies only a small fraction of the shell volume. The effect of the plumes on the overall flow is therefore reduced (Fig. 8d and f). The latitudinal temperature contrast between inside and outside the TC and thus the thermal winds around the TC (Fig. 8e) are weaker than those for $\eta=0.35$. The small inner core promotes simple structures of axisymmetric flow. The zonal flow outside the TC is strongly geostrophic.

\subsection{Fixed heat-flux boundary conditions}

The FF conditions at the outer boundary promote dipole-dominated stronger fields with Elsasser numbers between 3 and 11 as long as the local Rossby number remains below roughly 0.1. Axial dipole contributions clearly dominate the field at the outer boundary as well as throughout the shell. Consequently, $l=1$ and $m=0$ harmonics dominate the magnetic energy spectra at $R a^{\prime}=700$, shown in Fig. 3 (thick lines). Axisymmetric field contributions now carry as sizable fraction of the total magnetic energy: between $10 \%$ and $30 \%$ for $\eta=0.35$ and $30 \%$ and $44 \%$ for $\eta=0.10$. All this changes in the large Rayleigh number cases $R a^{\prime} \geq 6300$ with $R o_{\ell} \geq 0.120$ (see Table 1), where the dipole has lost its dominance and reverses more or less continuously.

Fig. 9 and 10 illustrate the spatial pattern of the field for $R a^{\prime}=700$. The 
radial field at the outer boundary shown in Fig. 9 demonstrates that the dipole dominates for both aspect ratios, but also that the azimuthal lengthscales are larger for $\eta=0.10$ than for $\eta=0.35$. This may in part be due to the lower degree of supercriticality in the case with a small inner core. However, comparisons for the mean wavenumbers of the flow and the magnetic field, $l_{\mathrm{u}}$ and $l_{\mathrm{B}}$, between cases with $\eta=0.35, R a^{\prime}=700$ and $\eta=0.10, R a^{\prime}=1400$, which have a similar degree of supercriticality, suggests that even under these conditions a larger inner core fosters smaller scales: $l_{\mathrm{B}}\left(l_{\mathrm{u}}\right)$ is $10.1(13.7)$ for $\eta=0.35, R a^{\prime}=700$ and $8.3(10.6)$ for $\eta=0.10, R a^{\prime}=1400$. Fig. 10b shows that the axisymmetric poloidal field lines are distorted inside the TC by the plume flow for $\eta=0.35$. This effect seems negligible for $\eta=0.10$ where the lines close to the rotation axis are nearly parallel to it (Fig. 10e). The toroidal fields shown in Fig. 10a and d also differ between the two cases. Two flux patches can be found in each hemisphere, one closer to the outer boundary near the equator and the other deeper inside the shell. The deep patches seem to be caused by an w-effect near the TC boundary, but they probably contribute little to the poloidal field production through an $\alpha$-effect because of the weak helicity in this region (see Fig. 10c and $\mathrm{f}$ ). These patches lie closer to the rotational axis for $\eta=0.10$ than for $\eta=0.35$.

Fig. 11 compares the axisymmetric temperature, zonal flow, and meridional circulation for the two FF cases at $R a^{\prime}=700$. The two solutions are similar but differ significantly from the FT cases shown in Fig. 8. In the FF case, the zonal flow shows strong variations along the $z$-direction (Fig. 11b and e). While Reynolds stress rules the zonal flow outside the TC in the FT solutions, thermal wind dominates in the FF cases. The pattern is similar to 
that of typical bottom heated dynamo simulations with strong dipolar magnetic fields. According to Aubert (2005), the associated Lorentz forces help to balance the Coriolis force and thereby partly release the Taylor-Proudman constraint and therefore allow for a large degree of $z$-dependence. Heat transport is more efficient in the equatorial region than in the regions closer to the poles. This can be quantified in terms of a co-latitude dependent Nusselt number:

$$
N u^{*}(\theta)=\frac{\Delta T_{\mathrm{S}}}{\Delta \bar{T}^{\phi}(\theta)}=\frac{T_{\mathrm{S}}\left(r_{\mathrm{i}}\right)-T_{\mathrm{S}}\left(r_{\mathrm{o}}\right)}{\bar{T}^{\phi}\left(r_{\mathrm{i}}, \theta\right)-\bar{T}^{\phi}\left(r_{\mathrm{o}}, \theta\right)},
$$

where the overbar with the suffix $\phi$ indicates the average over longitude. For the snapshots in Fig. $11 \mathrm{a}$ and $\mathrm{d}, N u^{*}(\pi / 2)=4.3$ and $N u^{*}(0)=2.1$ for $\eta=0.35, N u^{*}(\pi / 2)=2.7$ and $N u^{*}(0)=1.5$ for $\eta=0.10$. This latitudinal dependence in the convective heat transport results in a latitudinal temperature gradient in the FF cases. For the models at $R a^{\prime}=700$ depicted in Fig. $11 \mathrm{a}$ and $\mathrm{d}$, it amounts to 1.4 for $\eta=0.35$ and 2.3 for $\eta=0.10$, in terms of a normalized measure, $\left[\bar{T}^{\phi}\left(r_{\mathrm{o}}, \pi / 2\right)-\bar{T}^{\phi}\left(r_{\mathrm{o}}, 0\right)\right] / \bar{T}\left(r_{\mathrm{o}}\right)$. These latitudinal temperature gradients drive the strong thermal winds below the outer boundary in the equatorial region, shown in Fig. $11 \mathrm{~b}$ and e. The temperature distributions are also compliant with the large scale meridional circulation (Fig. 11c and $\mathrm{f}$ ), involving rising hot material around the equatorial plane and colder descending flow at the TC boundary. The meridional circulation contributes less than $1 \%$ to the kinetic energy, but is significantly stronger in the FF case than in the FT case (compare $M$ in Table 1). The effective magnetic Reynolds number for the meridional circulation, $R m^{\mathrm{M}}=R m \sqrt{M}$, is estimated to $4-45$. Since $R m^{\mathrm{M}}$ is larger than one, the circulation can affect the magnetic field. 
Fig. 12 illustrates contours of the $z$-components of the vorticity in the equatorial plane. A comparison with the equivalent illustration for the FT cases in Fig. 6 reveals that the flow is now much more large scale. The kinetic energy spectra with respect to spherical harmonic order $m$ show (thick solid lines in Figure 7) a dominance of low wavenumbers contributions in the FF cases, compared to the FT case. The mean degree $l_{\mathrm{u}}$ of the time-averaged spectra, listed in Table 1, confirms the increase in scale. For example, in the cases with $\eta=0.10$, we find $l_{\mathrm{u}}$ values between 9 and 12 for FF conditions, whereas it lies between 13 and 15 for FT conditions.

The effect of thermal boundary conditions on the onset of convection and finite-amplitude convection are examined by Takehiro et al. (2002), Busse \& Simitev (2006) and Gibbons et al. (2007). All three articles report that larger convective cells appear at the onset when FF conditions are employed at moderately small Ekman numbers. To explore whether the same effect is responsible for the differences found in our dynamo calculations, we also ran non-magnetic convection models with fixed flux at $R a^{\prime}=700$ for the two aspect ratios (listed in the lowest two rows of Table 1). Thick dotted lines in Figure $7 \mathrm{a}$ and $\mathrm{b}$ show the time-averaged kinetic energy spectra and Figure 13 illustrates the spatial pattern in the non-magnetic FF case at $\eta=0.10$. For both aspect ratios, non-magnetic convection shows flow structures that are similar to those found in the FT dynamos, for example, a geostrophic zonal wind and small-scale non-axisymmetric flow. We also find that the multipolar FF dynamo case with $R a^{\prime}=8400$ and $\eta=0.35$, which has a strong magnetic field with $\Lambda>7$, has similarly structures and length scales as the non-magnetic convection or dynamos with a fixed temperature condition. 
These observations suggest that a strong dipolar field is essential to maintain a balance between Lorentz force and Coriolis force that leads to the change of convective scale and differences in axisymmetric flow structures.

An interesting question is whether the differences in the flow between the non-magnetic FT and FF cases, even though they are weak, are directly causing the difference in the field morphology between FT and FF dynamos. If this is the case, the different field structure should appear, kinematically, in an early stage after a weak seed field is added on the non-magnetic convection. We tested this possibility for the FF case at $R a^{\prime}=700$ and $\eta=0.35$. Figure 14 shows the time evolution of the dipolarity, the field strength and the mean spherical harmonic degree of the flow. The initial seed field grows to a field of weak strength $(\Lambda \sim 0.3)$ and multipolar morphology $\left(f_{\text {dip }}^{\text {ax }} \sim 0.11\right)$ with a length scale $\left(l_{\mathrm{u}} \sim 17\right)$ similar to that for the non-magnetic stage, and stays in this state for approximately 1.3 magnetic diffusion time. After that, the field starts to increase in strength and dipolarity and the flow length scale becomes larger, settling finally at $\Lambda \sim 8.6, f_{\text {dip }}^{\text {ax }} \sim 0.65$ and $l_{\mathrm{u}} \sim 14$. This shows that the difference in flow structure, that is caused directly by the different thermal boundary conditions, is not sufficient to explain the different magnetic field morphologies. The slight change in the flow is sufficient to trigger, in the FF case, the growth of a dipolar field, however, it requires the back-reaction of this field on the flow structure for the dipole field to fully develop and reach its field strength. 


\section{Conclusion and Discussion}

In order to explore the early Martian dynamo and the early geodynamo, which both probably operated without an inner core being present, we conducted dynamo simulations purely driven by volumetric internal heating. Previous attempts in this direction have shown conflicting results concerning the magnetic field geometry. However, the simulations differed in the thermal boundary condition at the outer boundary and the size of an inner core that was retained for practical reason. Our simulations demonstrate that the discrepancies in these finding result from the thermal boundary conditions rather than the different inner core sizes. The fixed heat flux (FF) condition promotes dipole-dominated strong magnetic fields when the local Rossby number is not too large. As in the case of bottom-heated convection, the critical values for the local Rossby number are around 0.1. The condition of fixed temperature (FT) always yields multipolar and somewhat weaker fields, at least at an Ekman number of $10^{-4}$, irrespective of the inner core size. Numerical limitations prevented us from exploring inner cores with radii smaller than $10 \%$ of the total core radius, but the results by Aubert et al. (2009) for sizes down to $1 \%$ are consistent with our results. Note that the inner core size is more important for bottom-heated cases where its surface provides the main buoyancy sources (Heimpel et al., 2005).

In addition to the differences in the magnetic field, the FF and FT simulations also show profound differences in the flow structure. FF cases have thermal wind-dominated zonal flows, larger meridional circulation cells and a generally larger convective flow scale. This seems to be promoted by the stronger dipole field, which can more efficiently balance the Coriolis force. 
The resulting changes in the axisymmetric temperature distribution, the zonal wind structure and the meridional circulation have already been reported by Aubert (2005). The change towards larger length scales found in the non-axisymmetric pattern of the convective flow is an additional effect by the enhanced role of Lorentz forces associated with a dominantly dipolar field.

In the volume-heated cases we discuss here, the convective action and generation of magnetic field by helical motion is concentrated at larger radii than in the bottom-heated cases (compare Fig. 4 in Olson et al. (1999)). Therefore the inner core size has a minor influence while the outer thermal boundary condition has a profound impact. In the (predominantly) bottomheated cases, dipole-dominated fields are the norm for not too vigorous flow. In the volume-heated cases, the thermal boundary condition controls whether a dipolar or multipolar configuration is assumed.

The enlarged length scale in FF cases is reminiscent to the effects reported for magnetoconvection or Cartesian dynamo simulations where a strong enough imposed-magnetic field around $\Lambda=\mathcal{O}(1)$ promotes large and more vigorous flow structures (see Wicht et al. (2009) and references therein). The magnetoconvection simulations show a more drastic regime change than observed here from a weak-field branch with small-scale flow structures, whose typical wavenumbers are $\mathcal{O}\left(E k^{1 / 3}\right)$, to a strong-field branch with flow structures of $\mathcal{O}(1)$. They also demonstrate that the effect becomes larger for smaller Ekman numbers. Takahashi et al. (2008a) report a decrease in the mean harmonic degree of the flow, $l_{\mathrm{u}}$, of approximately $20 \%$ when comparing a dynamo simulation with a non-magnetic convection simulation at 
$E k=2 \times 10^{-6}$. This is the parameter range where magneto-convection calculations already see a much more drastic regime change. In our FF cases with $R a^{\prime}=700$ and $E k=10^{-4}$, the mean harmonic degree $l_{\mathrm{u}}$ in the FF dynamo case is approximately $25 \%$ lower than that in the non-magnetic FF convection case. A change in length scale comparable to that reported by Takahashi et al. (2008a) thus occurs also at larger Ekman number, $E k=10^{-4}$.

In our simulations the flow vigor is smaller in the FF cases. For example, we find $R m=204$ in the FT case at $R a^{\prime}=700$ and $\eta=0.35$ but only $R m=153$ in the respective FF simulation. This is not really in line with the picture of a weak field or a strong field branch, where a strong magnetic field not only increases the wavelengths of the flow but also the velocity. However, it agrees with the observation in many dynamo simulations that periods of stronger magnetic energy go along with reduced kinetic energy.

Sakuraba \& Roberts (2009) showed that the fixed heat flux condition promotes scale change in a simulation at Ekman number $E k \sim 2.4 \times 10^{-6}$ with a combination of internal and basal heating. They also claimed that the difference in scale between cases with fixed flux and fixed temperature exists only at low Ekman numbers. However, we find that the effect can already be observed at a much larger Ekman number of $E k=10^{-4}$. We confirmed the importance of the FF condition, which leads to a decrease of approximately $30 \%$ in mean harmonic degree $l_{\mathrm{u}}$ and a drastic increase of energy carried by low-order harmonics in the flow (Fig. 7). A difference is that we find multipolar fields when using a FT condition, whereas Sakuraba \& Roberts (2009) obtain a dipole-dominated field also in the FT case. The difference may be attributed to the lower Ekman number or the partial basal heating 
in their model.

It remains unclear how the different flow structures created by the thermal boundary conditions promote the different field morphologies. Results from kinematic dynamo studies propose that axial dipole parity is not favoured when the axisymmetric flow components are weak (e.g. Gubbins \& Gibbons, 2009). This does not agree with our findings where axial dipolar fields are compatible with rather small axisymmetric flows (compare our FF experiments, corresponding to $-0.12 \lesssim D \lesssim-0.05$ and $-0.005<M \lesssim-0.002$, with Fig. 1 or 11 in Gubbins \& Gibbons (2009)). The discrepancy between preferred parities in kinematic and the present MHD dynamos may suggest that mode selection is not explained only by linear kinematic process. Our simulation starting from non-magnetic convection with a small seed field, in which a strong dipolar field emerges only after a long transient stage with a weak multipolar field, shows that the back-reaction of the Lorentz forces modifying the flow plays an important role for selecting the field morphology. This mode selection seems to depend on a subtle balance, which the thermal boundary condition can tip one way as the other.

The mantle of terrestrial planets imposes a heat flux rather than a temperature on the boundary to the dynamo region. Fixed flux simulations are more appropriate than simulations with fixed temperature to model dynamos in terrestrial planets in general, the early Martian dynamo, and early geodynamo before the inner core developed in particular. Our simulations suggest that the early dynamos had dipole-dominated fields. 


\section{Acknowledgements}

K.H. has been supported partly by the Helmholtz Alliance 'Planetary

Evolution and Life'. Comments by two referees helped to improve the manuscript.

\section{References}

Aubert, J., 2005. Steady zonal flows in spherical shell dynamos. J. Fluid Mech., 542, 53-67.

Aubert, J., Labrosse, S., Poitou, C., 2009. Modelling the paleo-evolution of the geodynamo. Geophys. J. Int., 179, 1414-1428.

Busse, F.H., 2002. Convective flows in rapidly rotating spheres and their dynamo action. Phys. Fluid, 14, 1301-1314.

Busse, F.H., Simitev, R.D., 2006. Parameter dependences of convectiondriven dynamos in rotating spherical fluid shells. Geophys. Astrophys. Fluid Dyn., 100, 341-361.

Christensen, U.R., Aubert, J., 2006. Scaling properties of convection-driven dynamos in rotating spherical shells and application to planetary magnetic fields. Geophys. J. Int., 166, 97-114.

Christensen, U.R., Olson, P., Glatzmaier, G.A., 1999. Numerical modeling of the geodynamo: a systematic parameter study. Geophys. J. Int., 138, 393-409.

Christensen, U.R., Olson, P., 2003. Secular variation in numerical geodynamo models with lateral variations of boundary heat flow. Phys. Earth Planet. Int., 138, 39-54. 
Dormy, E., Soward, A.M., Jones, C.A., Jault, D., Cardin, P., 2004. The onset of thermal convection in rotating spherical shells. J. Fluid Mech., 501, 43-70.

Glatzmaier, G.A., 1984. Numerical simulations of stellar convective dynamos. I. The model and method. J. Comp. Phys., 55, 461-484.

Glatzmaier, G.A., Coe, R.S., Hongre, L.H., Roberts, P.H., 1999. The role of the Earth's mantle in controlling the frequency of geomagnetic reversals. Nature, 401, 885-890.

Gubbins, D., Gibbons, S.J., 2009. Kinematic dynamo action in a sphere with weak differential rotation. Geophys. J. Int., 177, 71-80.

Gibbons, S.J., Gubbins, D., Zhang, K., 2007. Convection in rotating spherical fluid shells with inhomogeneous heat flux at the outer boundary. Geophys. Astrophys. Fluid Dyn., 101, 347-370.

Grote, E., Busse, F.H., Tilgner, A., 2000. Regular and chaotic spherical dynamos. Phys. Earth Planet. Int., 117, 259-272.

Heimpel, M.H., Aurnou, J.M., Al-Shamali, F.M., Gomez Perez, N., 2005. A numerical study of dynamo action as a function of spherical shell geometry. Earth Planet. Sci. Lett., 236, 542-557.

Hollerbach, R., Jones, C.A., 1993. Influence of the Earth's inner core on geomagnetic fluctuations and reversals. Nature, 365, 541-543.

Jones, C.A., 2007. Thermal and compositional convection in the outer core. 
In: Olson, P. (Ed.), Core Dynamics, Treatise on Geophysics, Vol.8. Elsevier, Amsterdam, pp.131-185.

Kageyama, A., Sato, T., 1995. Computer simulation of a magnetohydrodynamic dynamo. II. Phys. Plasmas, 2, 1421-1431.

Kutzner, C., Christensen, U.R., 2000. Effects of driving mechanisms in geodynamo models. Geophys. Res. Lett., 27, 29-32.

Kutzner, C., Christensen, U.R., 2002. From stable dipolar towards reversing numerical dynamos. Phys. Earth Planet Int., 131, 29-45.

Labrosse, S., 2003. Thermal and magnetic evolution of the Earth's core. Phys. Earth Planet. Int., 140, 127-143.

Lillis, R.J., Frey, H.V., Manga, M., 2008. Rapid decrease in Martian crustal magnetization in the Noachian era: Implications for the dynamo and climate for early Mars. Geophys. Res. Lett., 35, L14203.

Nimmo, F., 2007. Thermal and compositional evolution of the core. In: Stevenson, D. (Ed.), Evolution of the Earth, Treatise on Geophysics, Vol.9. Elsevier, Amsterdam, pp.217-241.

Olson, P., Christensen, U.R., Glatzmaier, G.A., 1999. Numerical modeling of the geodynamo: Mechanisms of field generation and equilibration. J. Geophys. Res., 104, 10383-10404.

Olson, P., Christensen, U.R., 2002. The time-averaged magnetic field in numberical dynamos with non-uniform boundary heat flow. Geophys. J. Int., $151,809-823$. 
Olson, P., Christensen, U.R., 2006. Dipole moment scaling for convectiondriven planetary dynamos. Earth Planet. Sci. Lett., 250, 561-571.

Roberts, P.H., Glatzmaier, G.A., 2001. The geodynamo, past, present and future. Geophys. Astrophys. Fluid Dyn., 94, 47-84.

Sakuraba, A., Kono, M., 1999. Effects of the inner core on the numerical solution of the magnetohydrodynamic dynamo. Phys. Earth Planet. Int., $111,105-121$.

Sakuraba, A., Roberts, P.H., 2009. Generation of a strong magnetic field using uniform heat flux at the surface of the core. Nature Geoscience, 2, 802-805.

Stanley, S., Elkins-Tanton, L., Zuber, M.T., Parmentier, E.M., 2008. Mars' paleomagnetic field as the result of a single-hemispheredynamo. Science, $321,1822-1825$.

Stevenson, D.J., 2001. Mars' core and magnetism. Nature, 412, 214-219.

Stevenson, D.J., Spohn, T., Schubert, G., 1983. Magnetism and thermal evolution of the terrestrial planets. Icarus, 54, 466-489.

Takehiro, S., Ishiwatari, M., Nakajima, K., Hayashi, Y., 2002. Linear stability of thermal convection in rotating systems with fixed heat flux boundaries. Geophys. Astrophys. Fluid Dyn., 96, 439-459.

Takahashi, F., Matsushima, M., Honkura, Y., 2008a. Scale variability in convection-driven MHD dynamos at low Ekman number. Phys. Earth Planet. Int., 167, 168-178. 
Takahashi, F., Tsunakawa, H., Matsushima, M., Mochizuki, N., Honkura, Y., 2008b. Effects of thermally heterogeneous structure in the lowermost mantle on the geomagnetic field strength. Earth Planet. Sci. Lett., 272, 738-749.

Wicht, J., 2002. Inner-core conductivity in numerical dynamo simulations. Phys. Earth Planet. Int., 132, 281-302.

Wicht, J., Stellmach, S., Harder, H., 2009. Numerical models of the geodynamo: From fundamental Cartesian models to 3D simulations of field reversals. In: Glassmeier, K., Soffel, H., Negendank, J. (Eds.), Geomagnetic field variations, Springer Monograph. Springer-Verlag, Berlin Heidelberg, pp.107-158. 
Table 1: Dynamo cases with $E k=1 \times 10^{-4}, \operatorname{Pm}=3, \operatorname{Pr}=1$. In the second column labeled 'b.c.', FT and FF represent uniform temperature and heat flux boundary conditions, respectively. 'Morp.' indicates field morphologies. The fractions of axisymmetric parts of poloidal/toroidal kinetic $(M / D)$ and magnetic energies $(P / T)$ to total kinetic and magnetic energies, respectively, are represented in \%. The four last rows are for nonmagnetic convection cases with $E k=1 \times 10^{-4}, \operatorname{Pr}=1$. Cases highlighted by bold font are illustrated in Figures 4-13.

\begin{tabular}{|c|c|c|c|c|c|c|c|c|c|c|c|c|c|c|c|}
\hline$\eta$ & b.c. & $R a^{\prime}$ & $N u^{*}$ & $R m$ & $R o_{\ell}$ & $l_{\mathrm{u}}$ & $\Lambda$ & $f_{\mathrm{dip}}^{\mathrm{ax}}$ & $l_{\mathrm{B}}^{\mathrm{p}}$ & $m_{\mathrm{B}}^{\mathrm{p}}$ & Morp. & $M[\%]$ & $D[\%]$ & $P[\%]$ & $T[\%]$ \\
\hline 0.35 & $\mathrm{FT}$ & 350 & 1.6 & 136 & 0.024 & 16.5 & 0.06 & 0.052 & 3 & 1 & Octo. & 0.02 & 4.0 & 2.1 & 1.2 \\
\hline 0.35 & $\mathrm{FT}$ & 450 & 1.9 & 153 & 0.031 & 19.2 & 0.17 & 0.081 & $2-4$ & 2 & Hemis. & 0.02 & 4.1 & 3.1 & 1.3 \\
\hline 0.35 & FT & 700 & 2.5 & 204 & 0.043 & 19.6 & 0.48 & 0.085 & $3-5$ & 1 & Hemis. & 0.04 & 4.0 & 2.1 & 1.3 \\
\hline 0.35 & $\mathrm{FT}$ & 2100 & 4.9 & 372 & 0.078 & 19.8 & 1.95 & 0.091 & $7-15$ & $1-3$ & Multi. & 0.09 & 4.3 & 1.8 & 1.4 \\
\hline 0.35 & $\mathrm{FT}$ & 4200 & 7.1 & 511 & 0.110 & 20.2 & 4.02 & 0.075 & $9-20$ & $1-3$ & Multi. & 0.16 & 4.4 & 1.4 & 1.3 \\
\hline 0.10 & FT & 700 & 1.8 & 148 & 0.021 & 13.0 & 0.03 & 0.0002 & 2 & $0-1$ & Quad. & 0.02 & 7.3 & 13.1 & 6.2 \\
\hline 0.10 & $\mathrm{FT}$ & 1400 & 2.8 & 235 & 0.036 & 14.6 & 0.48 & 0.12 & $3-6$ & 1 & Hemis. & 0.03 & 5.4 & 4.3 & 2.3 \\
\hline 0.10 & $\mathrm{FT}$ & 2100 & 3.6 & 297 & 0.046 & 14.7 & 0.96 & 0.13 & $3-11$ & 1 & Hemis. & 0.05 & 5.0 & 3.6 & 2.3 \\
\hline 0.35 & $\mathrm{FF}$ & 450 & 2.1 & 119 & 0.016 & 12.1 & 5.64 & 0.72 & 1 & 0 & Dip. & 0.19 & 11.8 & 21.8 & 17.2 \\
\hline 0.35 & $\mathbf{F F}$ & 700 & 2.7 & 153 & 0.023 & 13.7 & 8.24 & 0.66 & 1 & 0 & Dip. & 0.27 & 9.6 & 18.2 & 13.5 \\
\hline 0.35 & $\mathrm{FF}$ & 2100 & 5.1 & 303 & 0.061 & 18.8 & 9.71 & 0.59 & 1 & 0 & Dip. & 0.23 & 4.9 & 10.5 & 5.1 \\
\hline 0.35 & $\mathrm{FF}$ & 4200 & 7.8 & 450 & 0.088 & 18.3 & 12.99 & 0.52 & 1 & 0 & Dip. & 0.30 & 7.0 & 5.7 & 4.5 \\
\hline 0.35 & $\mathrm{FF}$ & 6300 & 10.2 & 608 & 0.120 & 18.6 & 9.14 & 0.26 & $9-13$ & $0-1$ & Multi. & 0.36 & 13.1 & 3.4 & 4.0 \\
\hline 0.35 & $\mathrm{FF}$ & 8400 & 11.5 & 724 & 0.147 & 19.1 & 7.53 & 0.16 & 8-17 & 1 & Multi. & 0.39 & 11.9 & 2.3 & 2.3 \\
\hline 0.10 & FF & 700 & 2.0 & 123 & 0.012 & 9.4 & 3.52 & 0.83 & 1 & 0 & Dip. & 0.12 & 9.1 & 29.6 & 14.8 \\
\hline 0.10 & $\mathrm{FF}$ & 1400 & 3.1 & 181 & 0.021 & 10.6 & 8.49 & 0.62 & 1 & 0 & Dip. & 0.44 & 10.1 & 18.6 & 15.8 \\
\hline 0.10 & $\mathrm{FF}$ & 2100 & 3.9 & 230 & 0.028 & 11.2 & 11.01 & 0.59 & 1 & 0 & Dip. & 0.44 & 9.5 & 13.8 & 17.3 \\
\hline 0.10 & FT & 450 & 1.2 & 94 & 0.010 & 10.4 & - & - & - & - & No & 0.01 & 5.9 & - & - \\
\hline 0.10 & $\mathrm{FF}$ & 450 & 1.4 & 89 & 0.010 & 10.9 & - & - & - & - & No & 0.01 & 6.5 & - & - \\
\hline 0.35 & $\mathrm{FF}$ & 700 & 2.2 & - & 0.040 & 18.2 & - & - & - & - & - & 0.04 & 10.6 & - & - \\
\hline 0.10 & FF & 700 & 1.7 & - & 0.019 & 12.5 & - & - & - & - & $\square$ & 0.02 & 11.4 & - & - \\
\hline
\end{tabular}




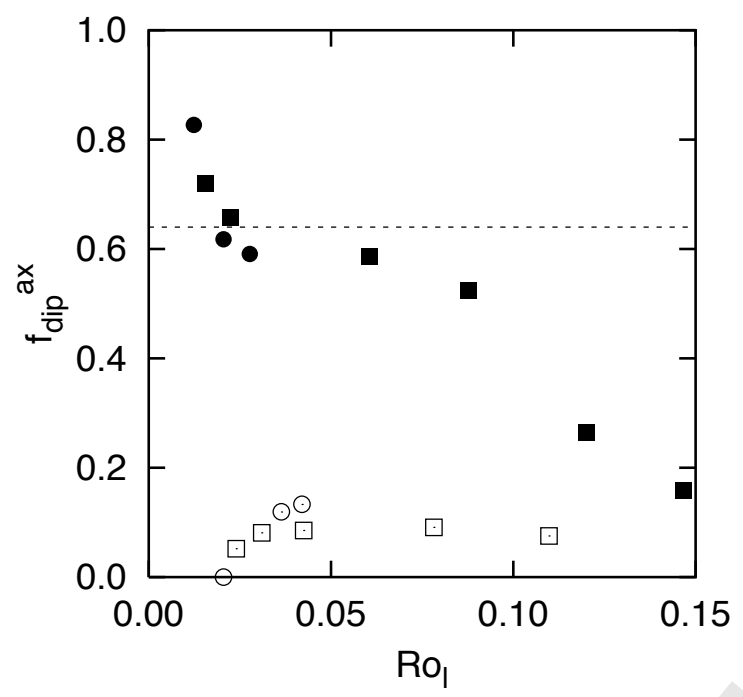

Figure 1: Relative axial-dipole strength versus local Rossby number. White and black symbols represent cases with fixed temperature (FT) and heat flux (FF) boundary conditions, respectively. The aspect ratio $\eta$ is indicated by the shapes of the symbols, 0.35 for squares and 0.10 for circles. The relative axial dipole strength of the present (2000) geomagnetic field is indicated by the broken line.
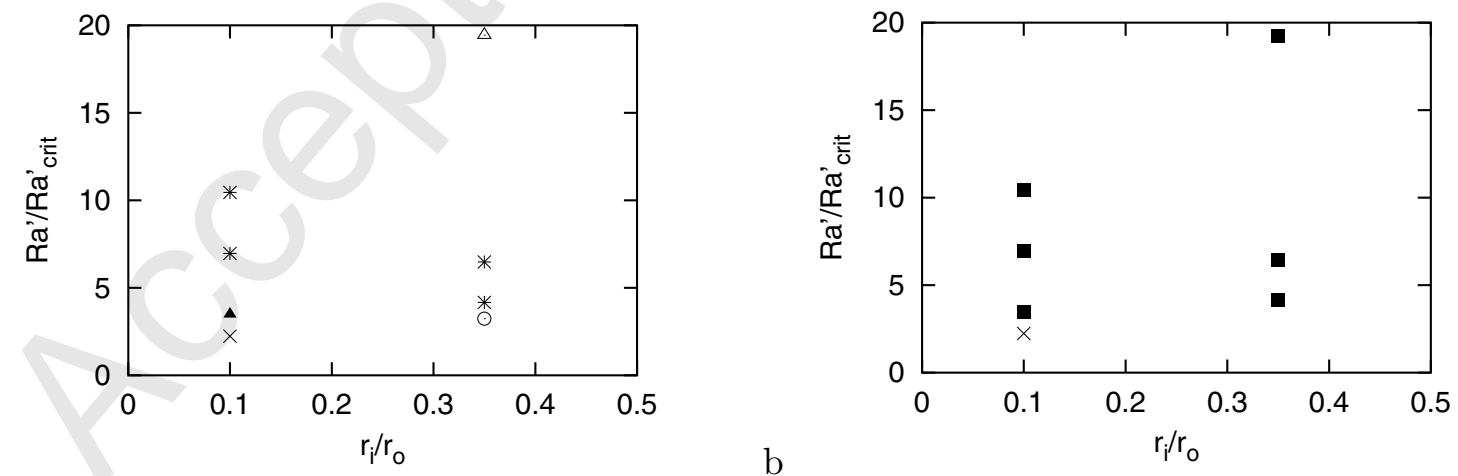

Figure 2: Phase diagrams for the field morphologies at $r=r_{\mathrm{O}}$ with FT (a) and FF (b) conditions. A filled square indicates axial dipolar field, a filled triangle - quadrupolar field, a circle - octopolar field, an asterisk - hemispherical field, an empty triangle - higher degree-dominated field and a cross - no dynamo. 

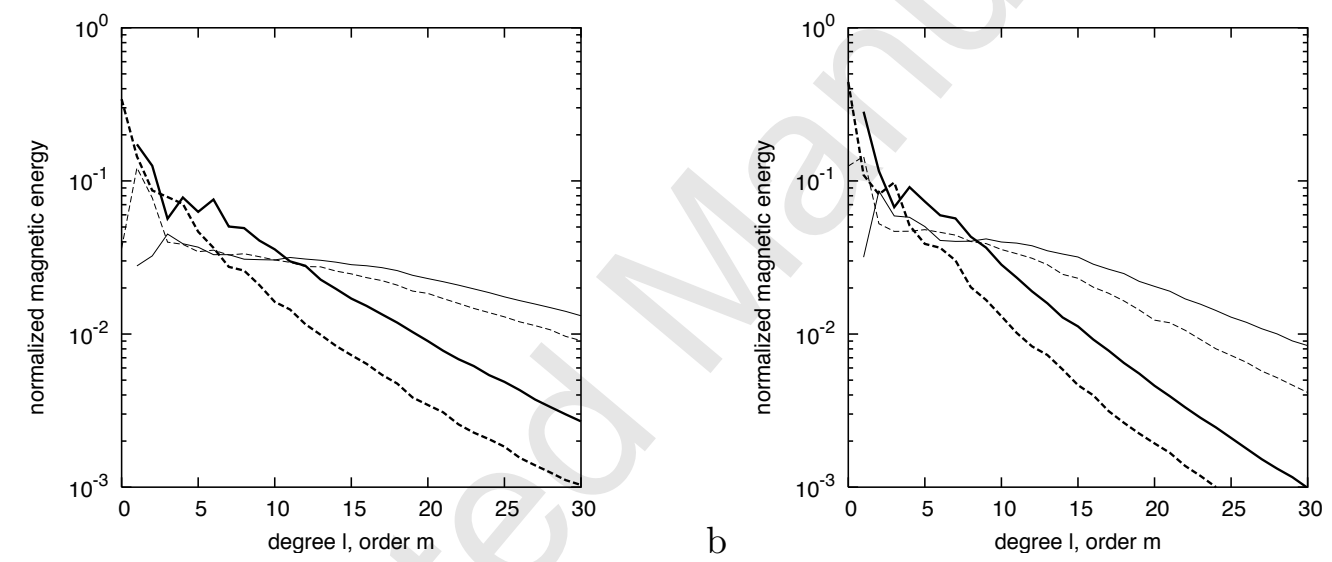

Figure 3: Magnetic spectra, averaged in time and radius and normalized by the total magnetic energy, for spherical harmonic degree $l$ (solid lines) and order $m$ (dashed lines) at $R a^{\prime}=700$ with aspect ratios $\eta=0.35$ (a) and 0.10 (b). Thin and thick lines represent cases for FT and FF conditions, respectively. 


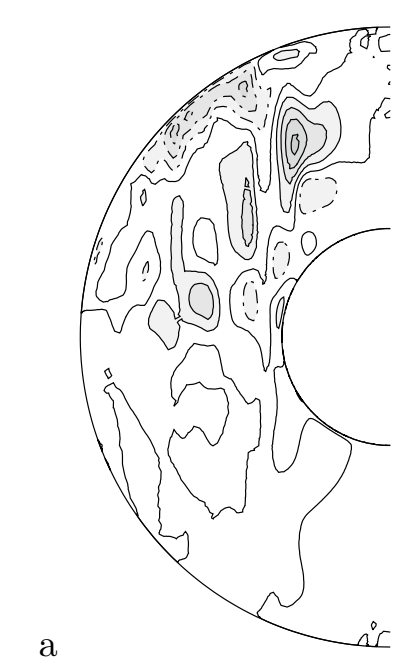

$\mathrm{b}$
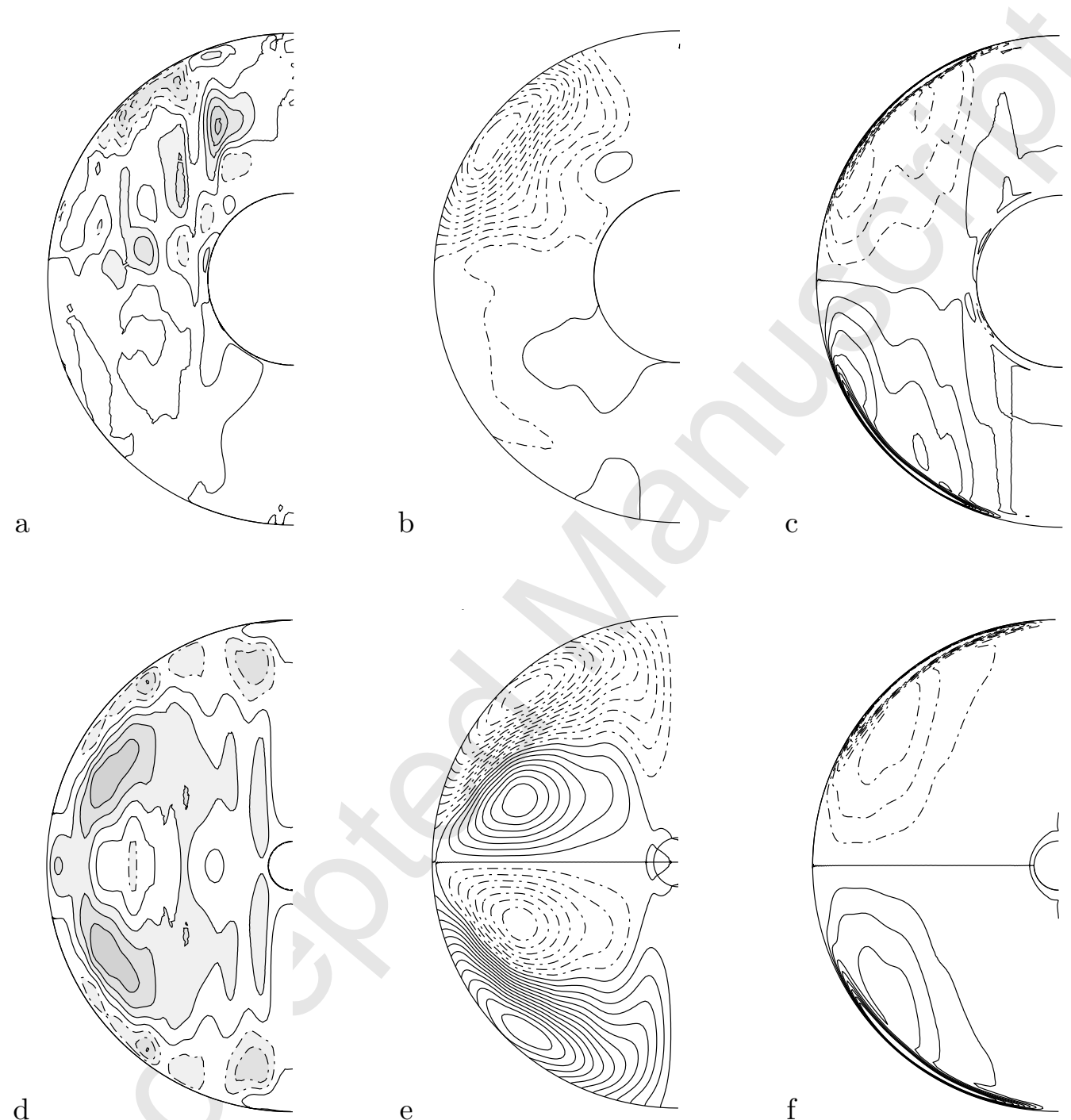

e

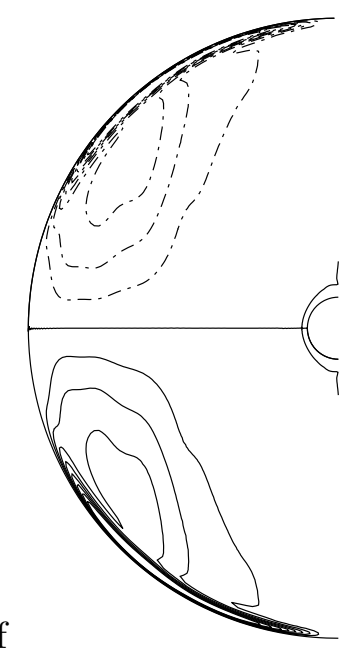

Figure 5: Axisymmetric parts of the toroidal field (a,d), poloidal field line (b,e) and helicity (c,f) for FT condition for $\eta=0.35$ (upper row, a-c) and 0.10 (lower row, d-f) with $R a^{\prime}=700$, at the same instant in time as in Figure 4a and b, respectively. Dash-dotted (solid) lines for negative (positive) values. Contour steps are 0.10 (a), 0.025 (d), 20000 (c) and 10000 (f). 


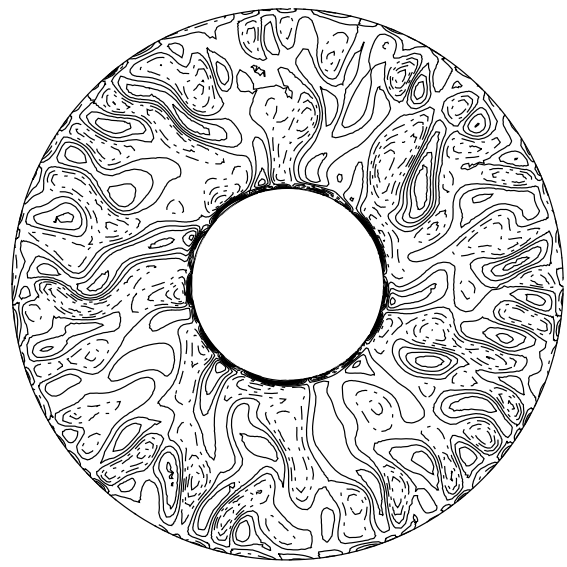

$\mathrm{b}$

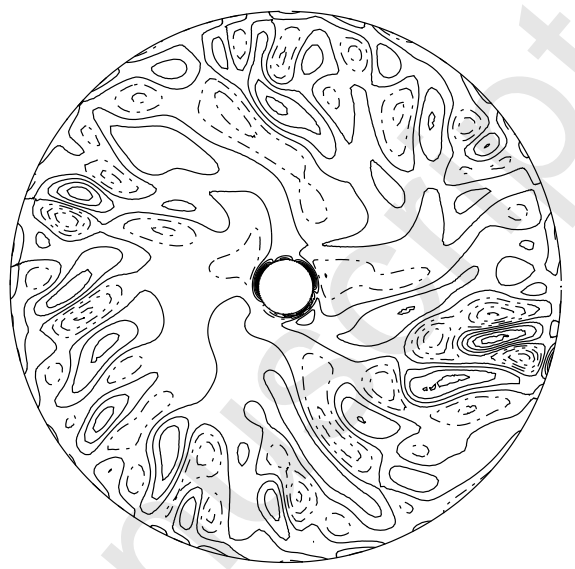

Figure 6: $\operatorname{Axial}(z)$ component of vorticity in the equatorial plane for FT condition at $\eta=0.35$ (a) and 0.10 (b) with $R a^{\prime}=700$ at the same instant in time as in Figure 4a and b, respectively. Solid lines for positive values. Contour interval is 600 .
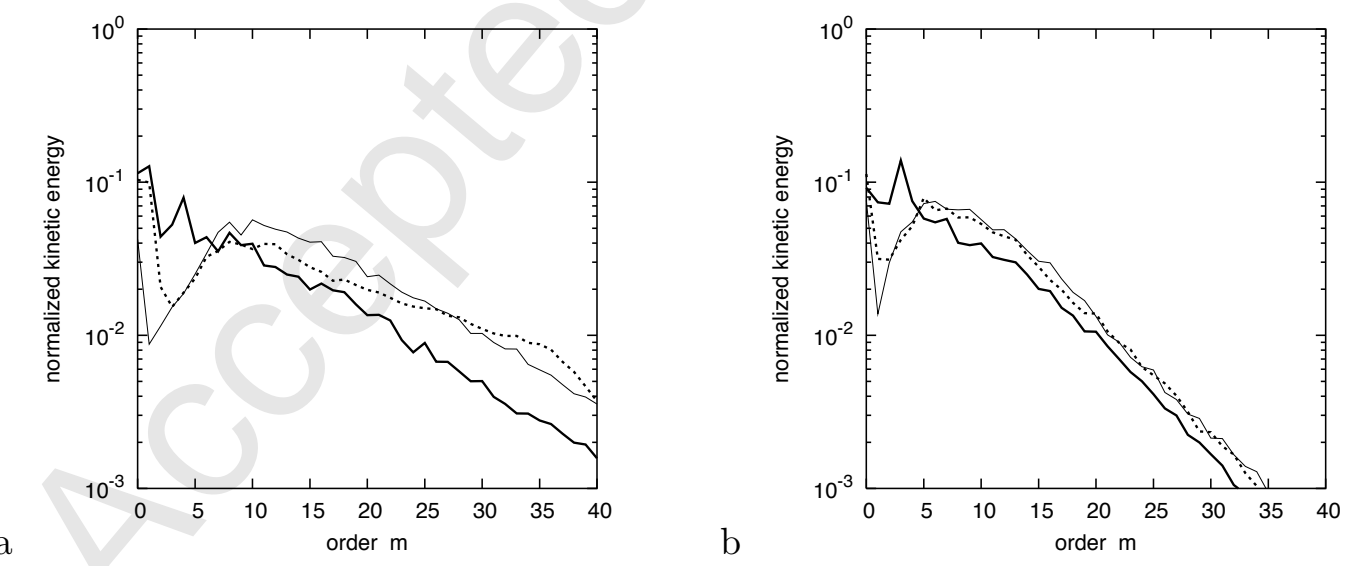

Figure 7: Time-averaged kinetic energy spectra as function of harmonic order, normalized by total kinetic energy, for $\eta=0.35$ (a) and 0.10 (b) with $R a^{\prime}=700$. Thin solid, thick solid and dotted lines represent the dynamo case with FT condition, the dynamo case with FF condition and the non-magnetic convection with FF condition, respectively. 

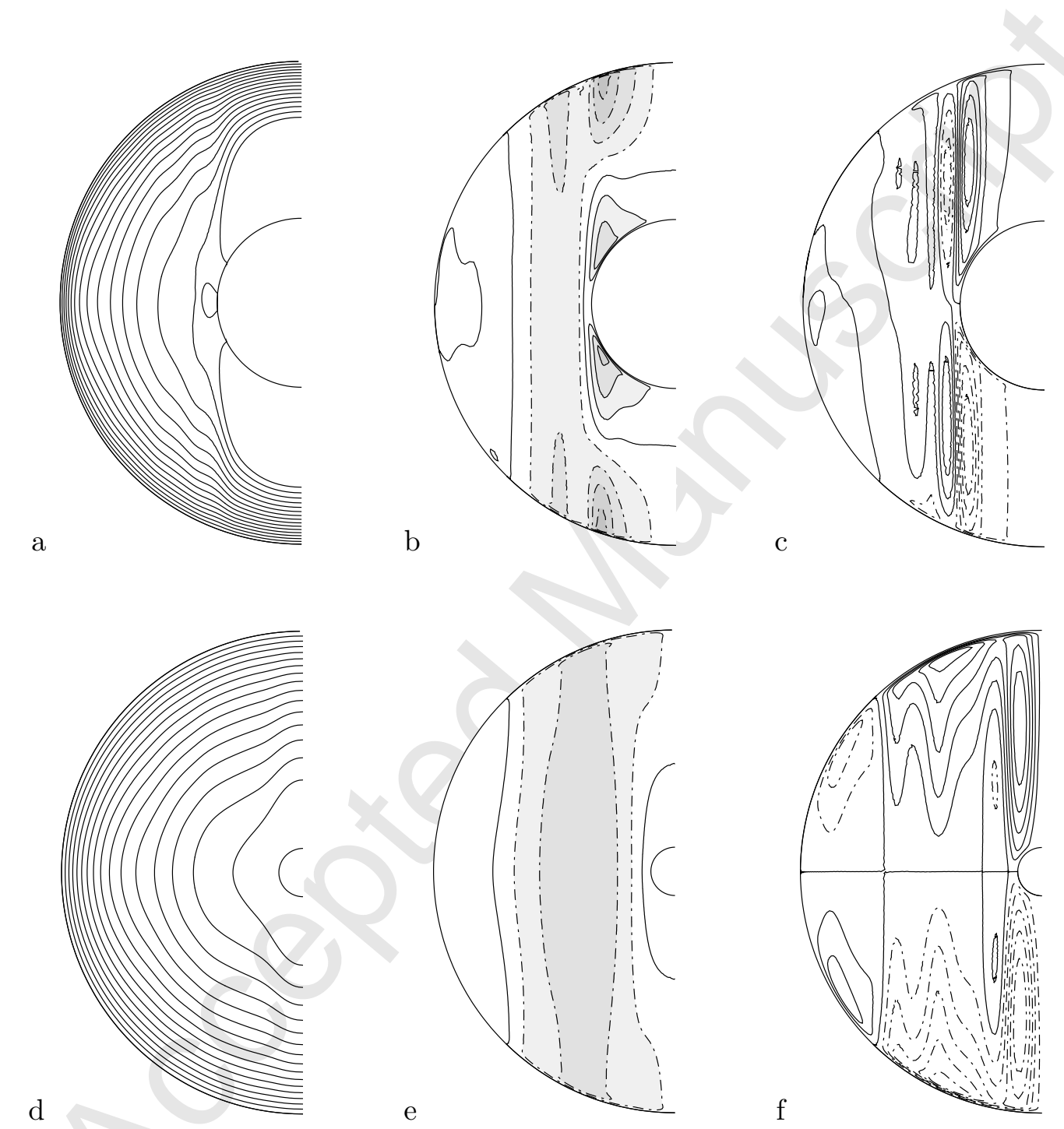

Figure 8: Axisymmetric parts of temperature $(\mathrm{a}, \mathrm{d})$, zonal $(\mathrm{b}, \mathrm{e})$ and meridional flow $(\mathrm{c}, \mathrm{f})$ for FT condition at $\eta=0.35$ (upper row, a-c) and 0.10 (lower row, d-f) with $R a^{\prime}=700$ at the same instant in time as in Figure 4a and b, respectively. Dashed lines for negative values and clockwise circulation, respectively. Contour steps are $0.02(\mathrm{a}, \mathrm{d})$ and $10(\mathrm{~b}, \mathrm{e})$. 
$\mathrm{a}$

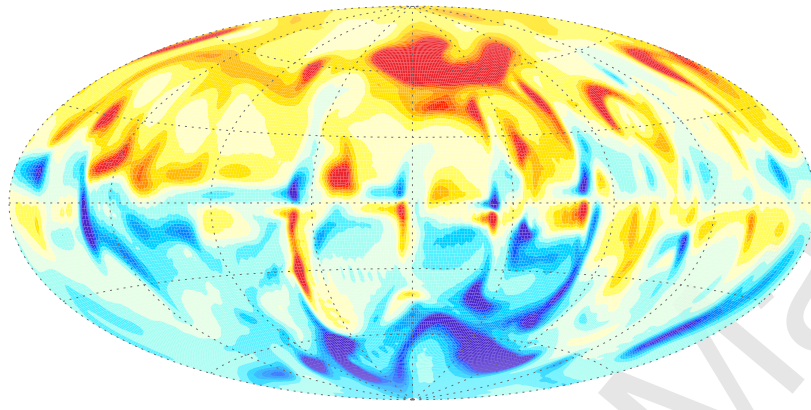

$\mathrm{b}$

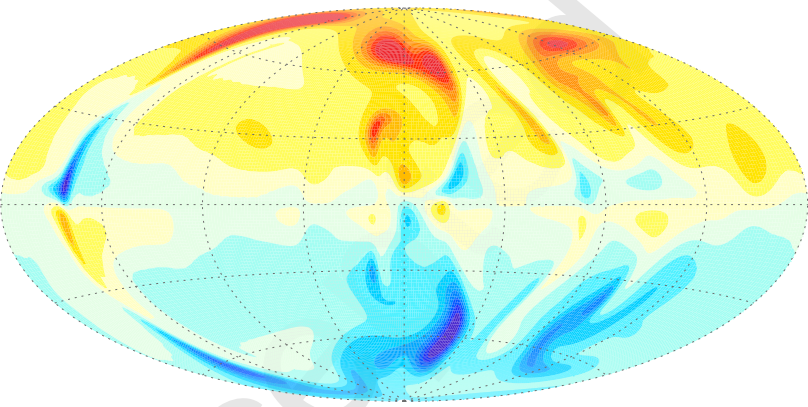

Figure 9: Radial component of the field at the outer boundary for FF condition at $R a^{\prime}=$ 700 with aspect ratio $\eta=0.35$ (a) and 0.10 (b). Contour interval is 0.25 . 

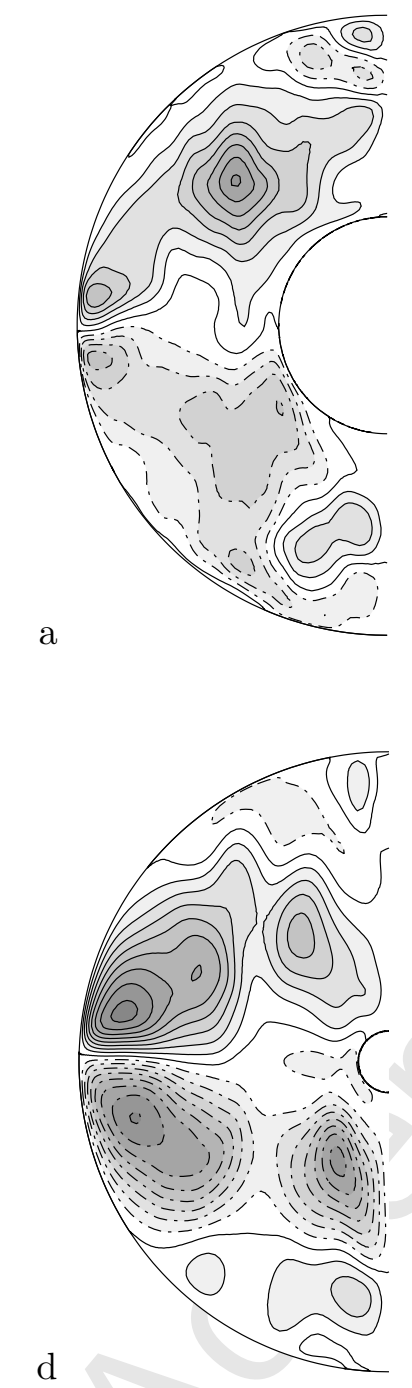

$\mathrm{b}$
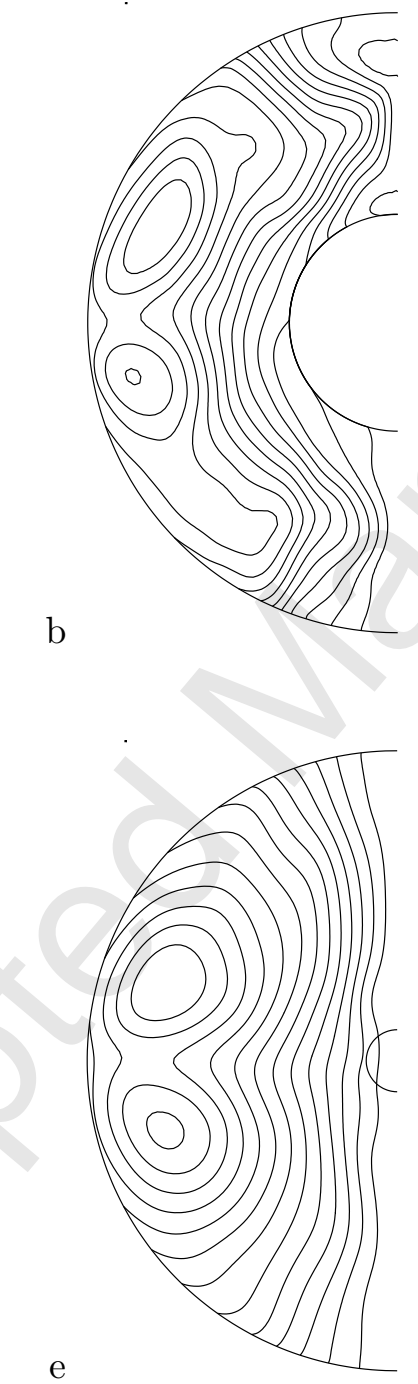

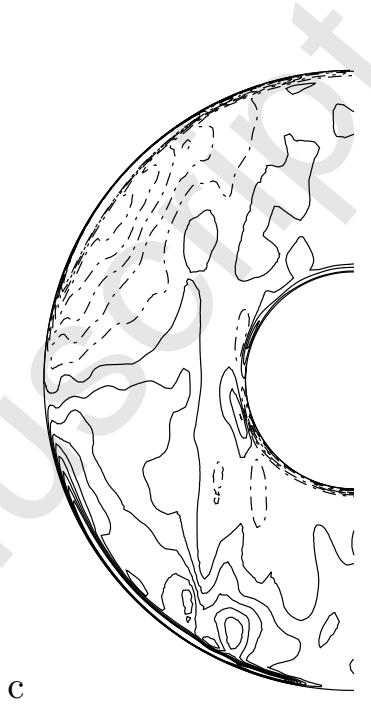

c

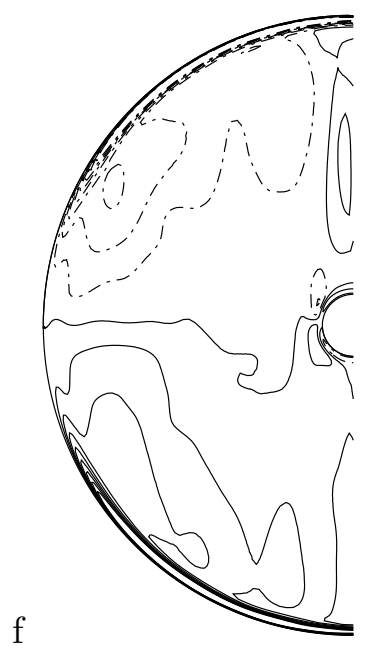

Figure 10: Axisymmetric parts of the toroidal field (a,d), poloidal field line (b,e) and helicity (c,f) for FF condition for $\eta=0.35$ (upper row, a-c) and 0.10 (lower row, d-f) with $R a^{\prime}=700$, at the same time as in Figure 9, respectively. Contour steps are 0.50 (a), 0.25 (d) and $5000(\mathrm{c}, \mathrm{f})$. 
a

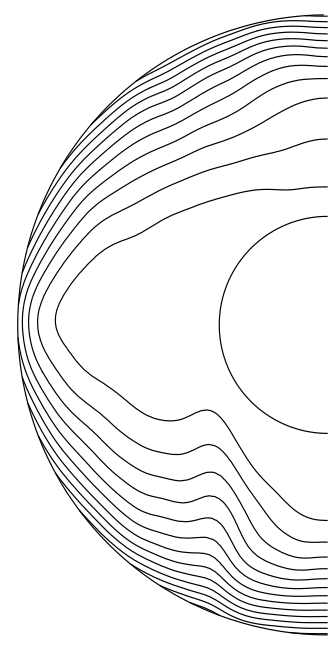

d

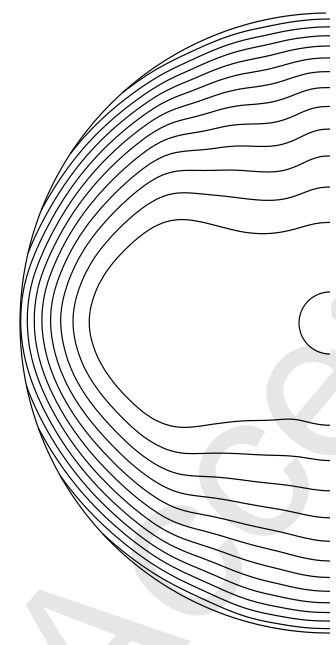

b

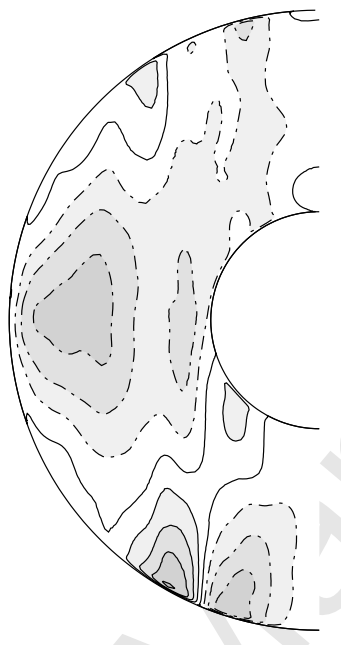

e

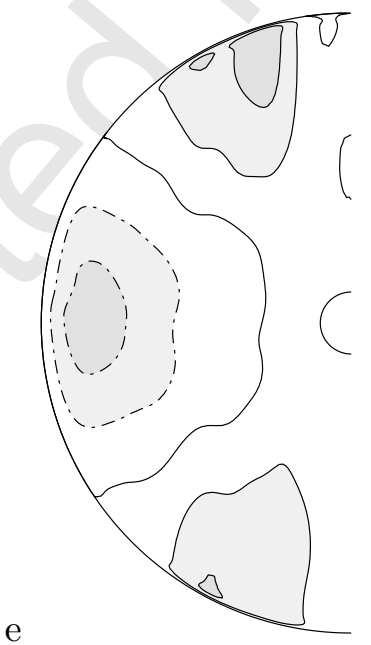

c

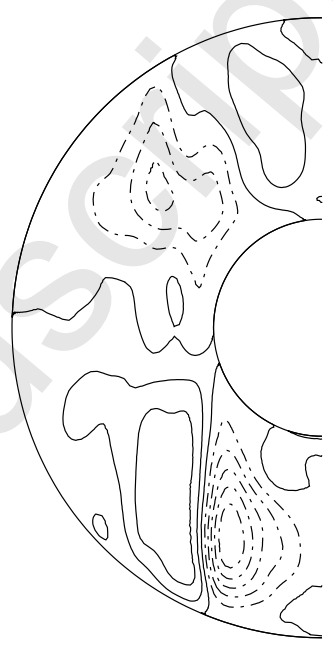

Figure 11: Axisymmetric temperature (a,d), zonal (b,e) and meridional flow (c,f) for FF condition at $\eta=0.35$ (upper row, a-c) and 0.10 (lower row, d-f) with $R a^{\prime}=700$ at the same time as in Figure 9a and b, respectively. Contour steps are 0.02 (a,d) and 10 (b,e). 
a

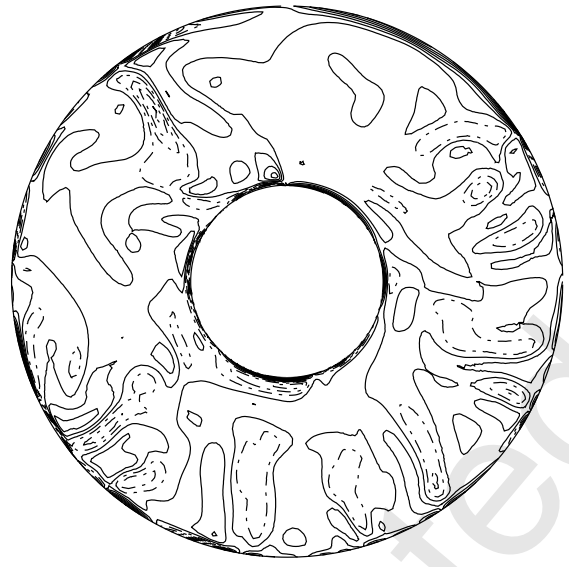

$\mathrm{b}$

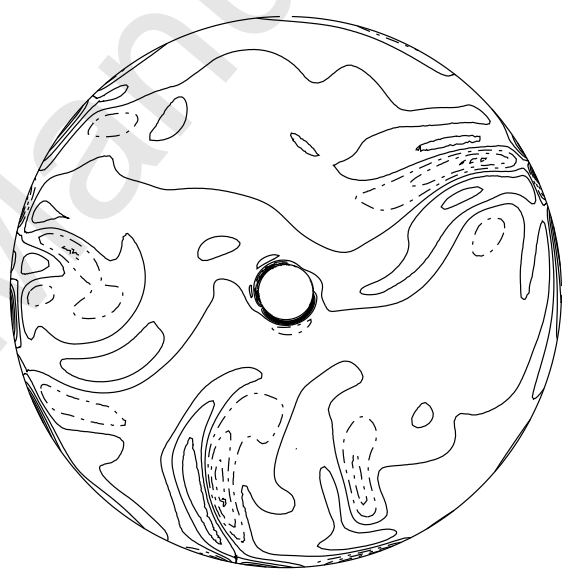

Figure 12: Axial(z)-component of vorticity in the equatorial plane for FF condition at $\eta=0.35$ (a) and 0.10 (b) with $R a^{\prime}=700$, at the same time as in Figure 9a and b, respectively. Contour step is 600 . 
a

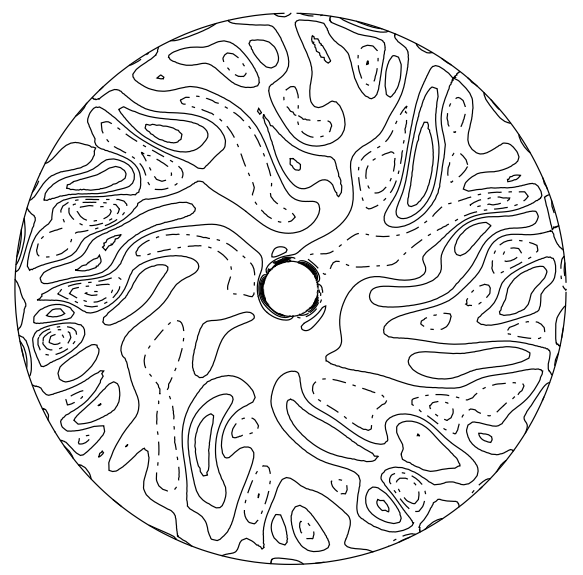

$\mathrm{b}$

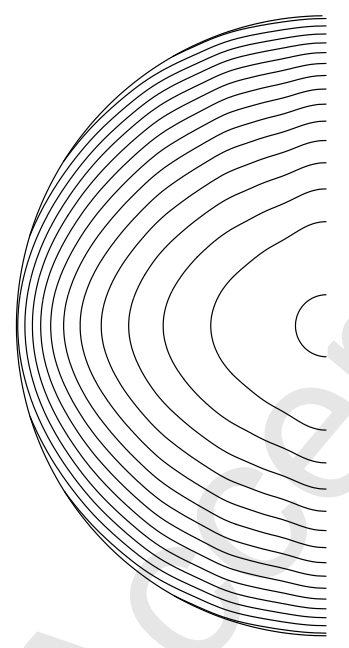

$\mathrm{c}$

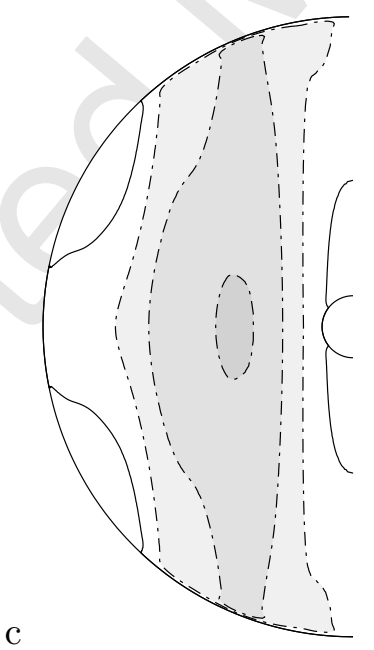

d

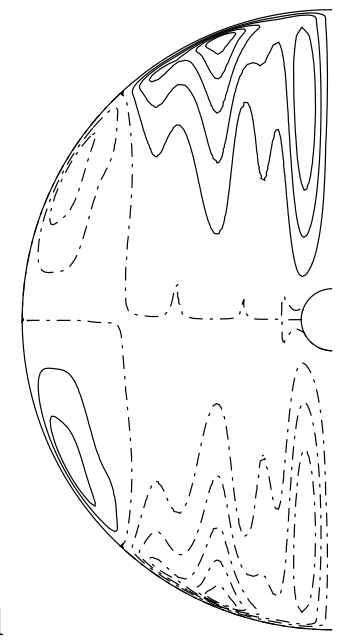

Figure 13: Non-magnetic convection with FF condition for $\eta=0.10$ and $R a^{\prime}=700$ : $z$-component of vorticity in the equatorial plane (a), axisymmetric temperature (b), zonal flow (c) and meridional flow (d). Contour steps are 600 (a), 0.02 (b) and 10 (c). 

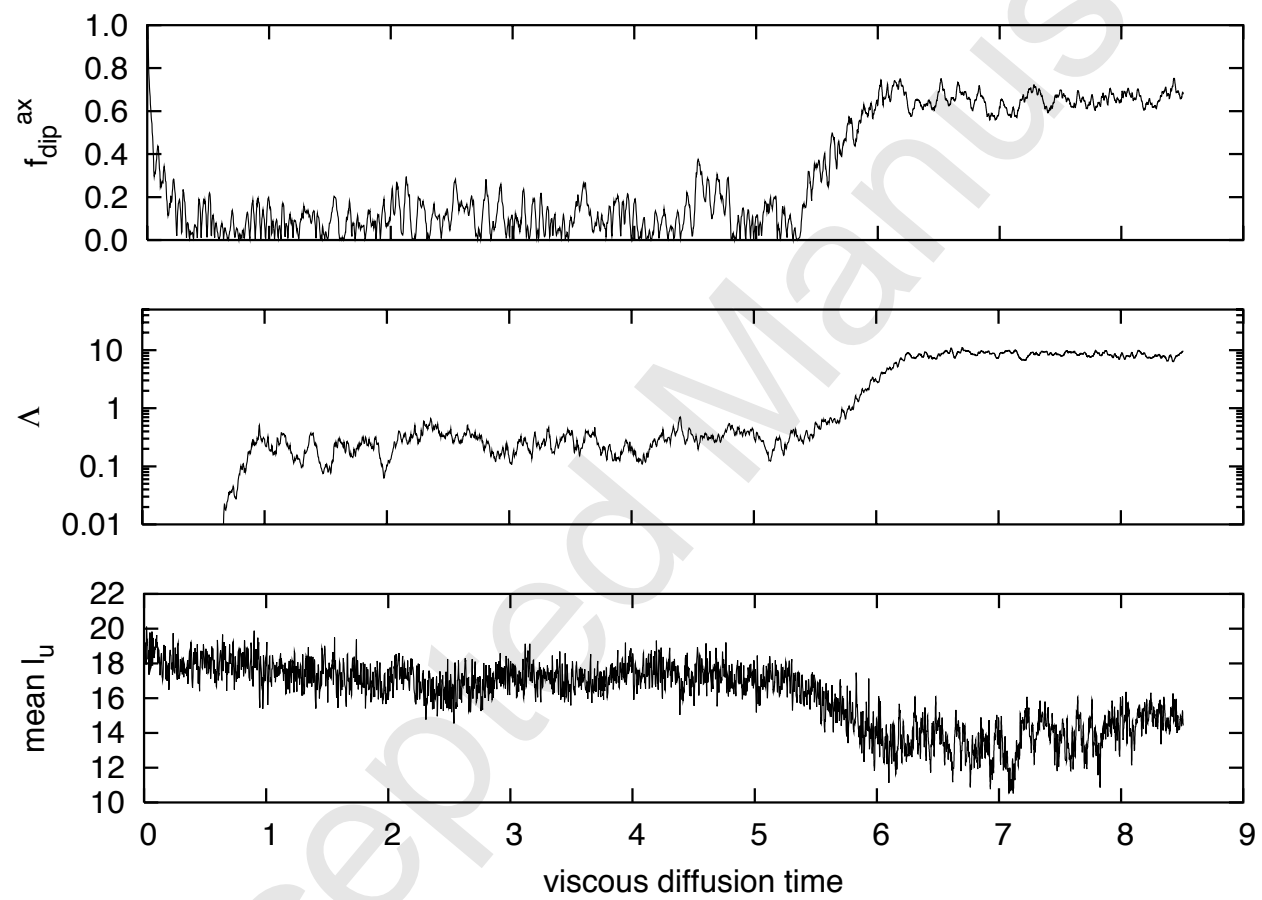

Figure 14: Dipolarity, Elsasser number and mean harmonic degree of flow (from top to bottom) versus time for $\mathrm{FF}$ dynamo case at $R a^{\prime}=700$ and $\eta=0.35$. 